This item was submitted to Loughborough's Research Repository by the author.

Items in Figshare are protected by copyright, with all rights reserved, unless otherwise indicated.

\title{
Envelope regularity discrimination
}

PLEASE CITE THE PUBLISHED VERSION

https://doi.org/10.1121/1.5100620

\section{PUBLISHER}

Acoustical Society of America

VERSION

$\mathrm{AO}$ (Author's Original)

\section{PUBLISHER STATEMENT}

This article may be downloaded for personal use only. Any other use requires prior permission of the author and the Acoustical Society of America. The following article appeared in Moore, BCJ; Sęk, AP; Vinay; Füllgrabe, C (2019): Envelope regularity discrimination. Journal of the Acoustical Society of America, 145(5), pp. $2861-2870$ and may be found at https://doi.org/10.1121/1.5100620

\section{LICENCE}

CC BY-NC-ND 4.0

\section{REPOSITORY RECORD}

Moore, BCJ, AP Sęk, Vinay, and Christian Fullgrabe. 2019. "Envelope Regularity Discrimination”. figshare. https://hdl.handle.net/2134/9438743.v1. 


\section{Envelope regularity discrimination}

Brian C. J. Moore, Aleksander P. Sęk, Vinay, and Christian Füllgrabe

Citation: The Journal of the Acoustical Society of America 145, 2861 (2019); doi: 10.1121/1.5100620

View online: https://doi.org/10.1121/1.5100620

View Table of Contents: https://asa.scitation.org/toc/jas/145/5

Published by the Acoustical Society of America

\section{ARTICLES YOU MAY BE INTERESTED IN}

The role of pitch and harmonic cancellation when listening to speech in harmonic background sounds The Journal of the Acoustical Society of America 145, 3011 (2019); https://doi.org/10.1121/1.5102169

Listening benefits in speech-in-speech recognition are altered under reverberant conditions

The Journal of the Acoustical Society of America 145, EL348 (2019); https://doi.org/10.1121/1.5100898

Personalized signal-independent beamforming for binaural hearing aids

The Journal of the Acoustical Society of America 145, 2971 (2019); https://doi.org/10.1121/1.5102173

Binaural unmasking with temporal envelope and fine structure in listeners with cochlear implants

The Journal of the Acoustical Society of America 145, 2982 (2019); https://doi.org/10.1121/1.5102158

The influence of distributed source regions in the formation of the nonlinear distortion component of cubic distortion-product otoacoustic emissions

The Journal of the Acoustical Society of America 145, 2909 (2019); https://doi.org/10.1121/1.5100611

A three-dimensional underwater sound propagation model for offshore wind farm noise prediction The Journal of the Acoustical Society of America 145, EL335 (2019); https://doi.org/10.1121/1.5099560

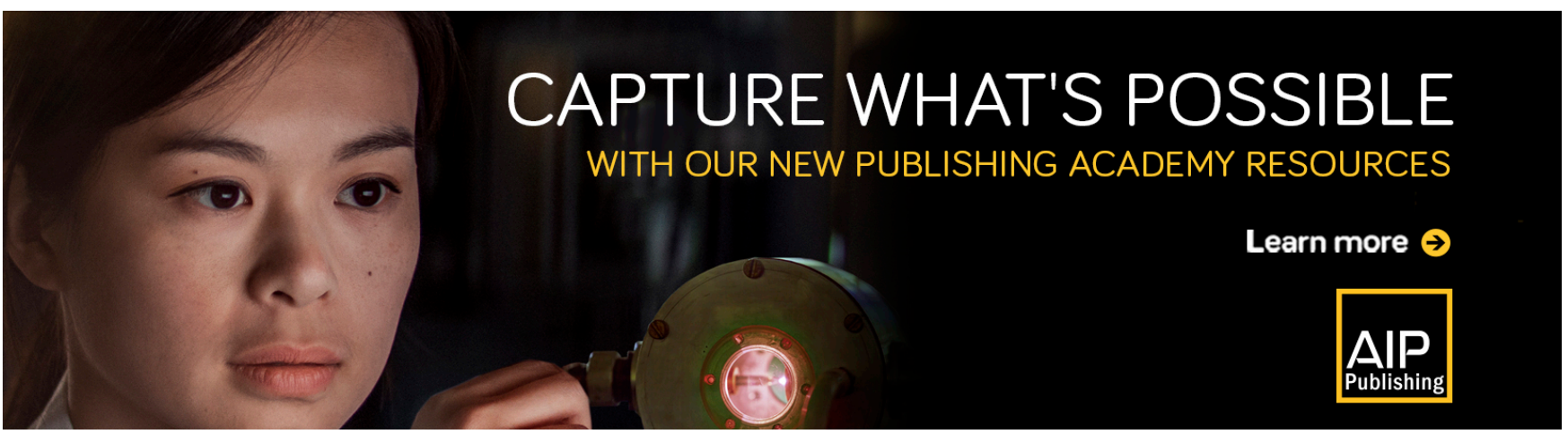




\title{
Envelope regularity discrimination
}

\author{
Brian C. J. Moore ${ }^{a)}$ \\ Department of Psychology, University of Cambridge, Downing Street, Cambridge CB2 3EB, United Kingdom
}

Aleksander P. Sęk

Institute of Acoustics, Faculty of Physics, Adam Mickiewicz University, Poznań, Poland

Vinay

Audiology Group, Department of Neuromedicine and Movement Science, Faculty of Medicine and Health

Sciences, Norwegian University of Science and Technology (NTNU), Tungasletta 2, Trondheim 7491, Norway

\author{
Christian Füllgrabe \\ School of Sport, Exercise and Health Sciences, Loughborough University, Ashby Road, Loughborough, \\ Leicestershire LE11 3TU, United Kingdom
}

(Received 30 January 2019; revised 11 April 2019; accepted 16 April 2019; published online 8 May 2019)

\begin{abstract}
The ability to discriminate irregular from regular amplitude modulation was assessed using the "envelope regularity discrimination" test. The amount of irregularity was parametrically varied and quantified by an "irregularity index." Normative data were gathered for young subjects with normal audiometric thresholds. Parameters varied were the carrier and modulation frequencies, $f_{c}$ and $f_{m}$, and the baseline modulation index, $m$. All tests were performed using a background thresholdequalizing noise. The main findings were (1) using $f_{c}=4000 \mathrm{~Hz}, f_{m}=8 \mathrm{~Hz}$, and $m=0.3$, performance improved over the first two threshold runs and then remained roughly stable, and there was a high correlation between thresholds obtained at $80 \mathrm{~dB}$ sound pressure level (SPL) and at $20 \mathrm{~dB}$ sensation level; (2) using $f_{m}=8 \mathrm{~Hz}$ and $m=0.3$ with a level of $80 \mathrm{~dB}$ SPL, thresholds did not vary significantly across $f_{c}=1000,2000$, and $4000 \mathrm{~Hz}$; (3) using $f_{m}=8 \mathrm{~Hz}$ and $f_{c}=4000 \mathrm{~Hz}$ with a level of $80 \mathrm{~dB}$ SPL, thresholds did not vary significantly for $m$ from 0.2 to 0.5 ; and (4) using $m=0.3$ and $f_{c}=4000 \mathrm{~Hz}$ with a level of $80 \mathrm{~dB}$ SPL, thresholds improved with increasing $f_{m}$ from 2 to $16 \mathrm{~Hz}$. For all conditions, there was substantial individual variability, probably resulting from differences in "processing efficiency." (C) 2019 Acoustical Society of America.
\end{abstract}

https://doi.org/10.1121/1.5100620

[AKCL]

Pages: 2861-2870

\section{INTRODUCTION}

Broadband sounds, such as speech and music, are decomposed in the cochlea into a series of narrowband sounds, each of which can be considered as a slowly varying envelope superimposed on a rapidly fluctuating carrier, often called the temporal fine structure (Moore, 2014). It is widely believed that the envelope fluctuations in different frequency channels convey important information for speech perception (Plomp, 1983; Shannon et al., 1995). Abnormalities in envelope processing may contribute to the difficulties experienced by hearing-impaired and older people in understanding speech (Moore and Glasberg, 1993; Ruggles et al., 2012; Füllgrabe et al., 2015; Schlittenlacher and Moore, 2016). There have been many studies of the ability of normalhearing and hearing-impaired subjects to detect amplitude modulation (AM) as a function of modulation rate (Viemeister, 1979; Bacon and Viemeister, 1985; Bacon and Gleitman, 1992; Moore et al., 1992) and a few studies of the ability to detect changes in AM depth (Wakefield and Viemeister, 1990; Schlittenlacher and Moore, 2016) and changes in AM rate (Burns and Viemeister, 1976; Patterson

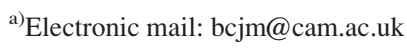

and Johnson-Davies, 1977; Lemanska et al., 2002). However, to our knowledge, there are no published studies of the ability to discriminate regular AM from irregular AM. The present paper describes a test of envelope regularity discrimination, called the ERD test, and presents outcomes of the test for normal-hearing subjects aged 41 years or less.

One motivation behind the development of the ERD test stems from recent work using rodents, which has shown that exposure to sounds that produce a temporary threshold shift can lead to loss of synaptic ribbons between the inner hair cells and the neurons in the cochlea (Kujawa and Liberman, 2009, 2015; Liberman and Kujawa, 2017). Effectively, this synaptopathy disconnects the neurons in the auditory nerve from the cochlea, leading to a reduced flow of information along the auditory nerve. Following the synaptopathy, the disconnected neurons themselves may degenerate. Rodents seem to be more susceptible to synaptopathy than humans (Dobie and Humes, 2017), but synaptopathy has been observed in primates following intense noise exposure (Valero et al., 2017). Synaptopathy also occurs with increasing age in both animals and humans (Sergeyenko et al., 2013; Viana et al., 2015). It has been suggested that synaptopathy contributes to difficulties in understanding speech in difficult listening situations, for example, when background sounds and/or reverberation are present (Liberman et al., 
2016; Liberman and Kujawa, 2017). Synaptopathy and other forms of auditory dysfunction combined with a normal audiogram have been called "hidden hearing loss" (Schaette and McAlpine, 2011). However, in the present paper the term "hidden hearing disorder" (HHD) is used, since the term "hearing loss" is traditionally associated with elevated audiometric thresholds.

The discovery of synaptopathy in animal models has led to a search for comparable effects in humans with normal audiograms. Both electrophysiological and psychoacoustical measures have been used in attempts to determine when synaptopathy is present. However, the results have been mixed. Some studies have revealed differences between nonexposed and noise-exposed groups in electrophysiological, psychoacoustical, and/or speech perceptual measures (Liberman et al., 2016; Bramhall et al., 2017; Grose et al., 2017), but some studies have failed to find such effects (Prendergast et al., 2017a; Prendergast et al., 2017b; Yeend et al., 2017; Guest et al., 2018b; Valderrama et al., 2018). Because synaptopathy is likely to be more severe when there are also other forms of cochlear damage, such as outer hair cell $(\mathrm{OHC})$ damage, there is a need to find tests that reveal the presence of synaptopathy when the audiogram is not normal.

Several researchers have measured AM detection thresholds in an attempt to detect subtle auditory dysfunction, including HHD (Stone and Moore, 2014; Bharadwaj et al., 2015). It has been assumed that higher (poorer) AM detection thresholds are associated with increasing HHD. However, there are some problems associated with this measure, especially when testing people with elevated audiometric thresholds. First, AM detection thresholds may not be sufficiently sensitive to "noise" in the auditory coding of AM caused by HHD (Oxenham, 2016). Second, OHC dysfunction can improve the ability to detect AM (Jerger, 1962; Füllgrabe et al., 2003; Ernst and Moore, 2012; Schlittenlacher and Moore, 2016), probably because of the loss of cochlear compression (Moore et al., 1996). This may offset deleterious effects of the loss of synapses and/or neurons on AM detection.

The ERD test described in this paper suffers less from these problems. The ERD test provides a measure of the ability to discriminate regular sinusoidal AM of a sinusoidal carrier from $\mathrm{AM}$ of the same carrier that is irregular in rate and amount. The degree of irregularity is adaptively varied to determine the smallest irregularity that can be detected. The ERD test is intended to reveal auditory conditions that lead to reduced precision of coding of the envelopes of sounds in the auditory system. If the representation of envelopes in the auditory system is "noisy," then regular AM may sound somewhat irregular in rate and/or amount. This should lead to worse performance on the ERD test. The ERD test involves stimuli with clearly audible AM depths. Loudness recruitment caused by reduced OHC function may magnify the perceived AM depth (Moore et al., 1996). The effect is similar to multiplying the AM depth by a certain factor. Assuming that performance of the ERD test depends on the amount of envelope irregularity relative to the rootmean-square (RMS) modulation depth, loudness recruitment should have little or no influence on performance of the task. This argument is presented in more detail in Sec. II. To assess whether performance of the ERD test did indeed depend on the amount of envelope irregularity relative to the RMS modulation depth, one experiment of the present paper assessed performance on the ERD test as a function of the modulation depth of the stimuli (which was matched for the regular and irregular AM). Performance was also assessed as a function of level, carrier frequency, ear of presentation, and rate of modulation.

\section{DESIGN OF THE ERD TEST}

A two-alternative forced-choice (2AFC) task was used. There were two observation intervals separated by $200 \mathrm{~ms}$. Each interval contained an amplitude-modulated sinusoidal carrier lasting $1000 \mathrm{~ms}$, including 20-ms raised-cosine rise/ fall ramps. In one randomly chosen interval, called the nonsignal interval, the AM was perfectly regular sinusoidal AM. In the other interval, called the signal interval, the AM was irregular in rate and amount, i.e., the modulator was noiselike. The starting phase of the modulator was randomly chosen for each interval. The subject was asked to pick the interval in which the AM sounded irregular. Trial-by-trial feedback was provided on a screen in front of the subject, to help them to "learn what to listen for."

The amount of irregularity was specified by the "irregularity index," II. The greater the value of the II, the greater was the irregularity. The II was defined in the following way. The spectrum of the stimulus in the non-signal interval had a component at the carrier frequency, $f_{c}$, and two sidebands at $f_{c}-f_{m}$ and $f_{c}+f_{m}$, where $f_{m}$ is the modulation frequency. For example, if $f_{c}=4000 \mathrm{~Hz}$ and $f_{m}=8 \mathrm{~Hz}$, the sidebands fell at 3992 and $4008 \mathrm{~Hz}$. All components in the non-signal stimulus had a starting phase of $0^{\circ}$. The amplitudes of the sidebands relative to the amplitude of the carrier were determined by the modulation index, $m$. For example, if $m=0.5$, each sideband had an amplitude that was 0.25 times the amplitude of the carrier. The amplitude of each sideband relative to the amplitude of the carrier is denoted $A_{n s}$. The spectrum of the stimulus in the signal interval contained the same frequency components as for the non-signal interval, but the relative amplitude of the two sidebands $\left(A_{s}\right)$ was lower; $A_{s}<A_{n s}$. In addition the spectrum contained components with frequencies spaced at $1-\mathrm{Hz}$ intervals from $f_{c}-f_{m}+1$ to $f_{c}+f_{m}-1 \mathrm{~Hz}$. For example, if $f_{c}=4000 \mathrm{~Hz}$ and $f_{m}=8 \mathrm{~Hz}$, the additional components fell at 3993, 3994, 3995, 3996, 3997, 3998, 3999, 4001, 4002, 4003, 4004, 4005, 4006, and $4007 \mathrm{~Hz}$. The phases of these additional components were chosen randomly for each trial. These components all had the same amplitude relative to that of the $4000-\mathrm{Hz}$ component. This relative amplitude is denoted $B$. The ratio $B / A_{s}$ is the II. When the II is large, there is more irregularity. When the II is small, there is less irregularity. In what follows, the II is expressed in decibels as $20 \log _{10}\left(B / A_{s}\right)$. Note that the II expresses the amount of irregularity relative to the baseline modulation index $m$.

For each value of the II, the AC-component of the envelope of the waveform for the signal interval was scaled by 
adjusting $B$ and $A_{s}$ by the same factor so as to equate its RMS value to that for the AC-component of the envelope in the non-signal interval. In addition, the RMS value of the waveform for the signal interval was scaled so that it matched the RMS value for the non-signal interval. Figure 1 shows examples of the envelopes of stimuli with $m=0.3$ (left) and $m=0.5$ (right) for three values of the II. The envelopes are plotted on a $\mathrm{dB}$ scale. A fully modulated carrier with no irregularity $(m=1)$ would have a peak envelope magnitude of $0 \mathrm{~dB}$. Note that the envelopes are irregular in both AM depth and AM rate. Thus, the ERD test does not distinguish the ability to detect irregularities in AM depth and AM rate, although it seems likely that the irregularities in AM depth are more important. Note also that for a given II, the amount of irregularity increases as $m$ increases.

Performance of the ERD test is probably based mainly on the fact that the peak-to-valley ratio (PVR, expressed in $\mathrm{dB}$ ) of the envelope varies from one modulation cycle to the next for the signal stimulus, while this is not the case for the non-signal stimulus. The variability in PVR can be quantified using its standard deviation, $\mathrm{PVR}_{\mathrm{SD}}$. It seems plausible to assume that the threshold in the ERD test corresponds to a certain ratio of $\mathrm{PVR}_{\mathrm{SD}}$ to the mean PVR, $\mathrm{PVR}_{\text {mean }}$ (which is approximately the same for the signal and non-signal
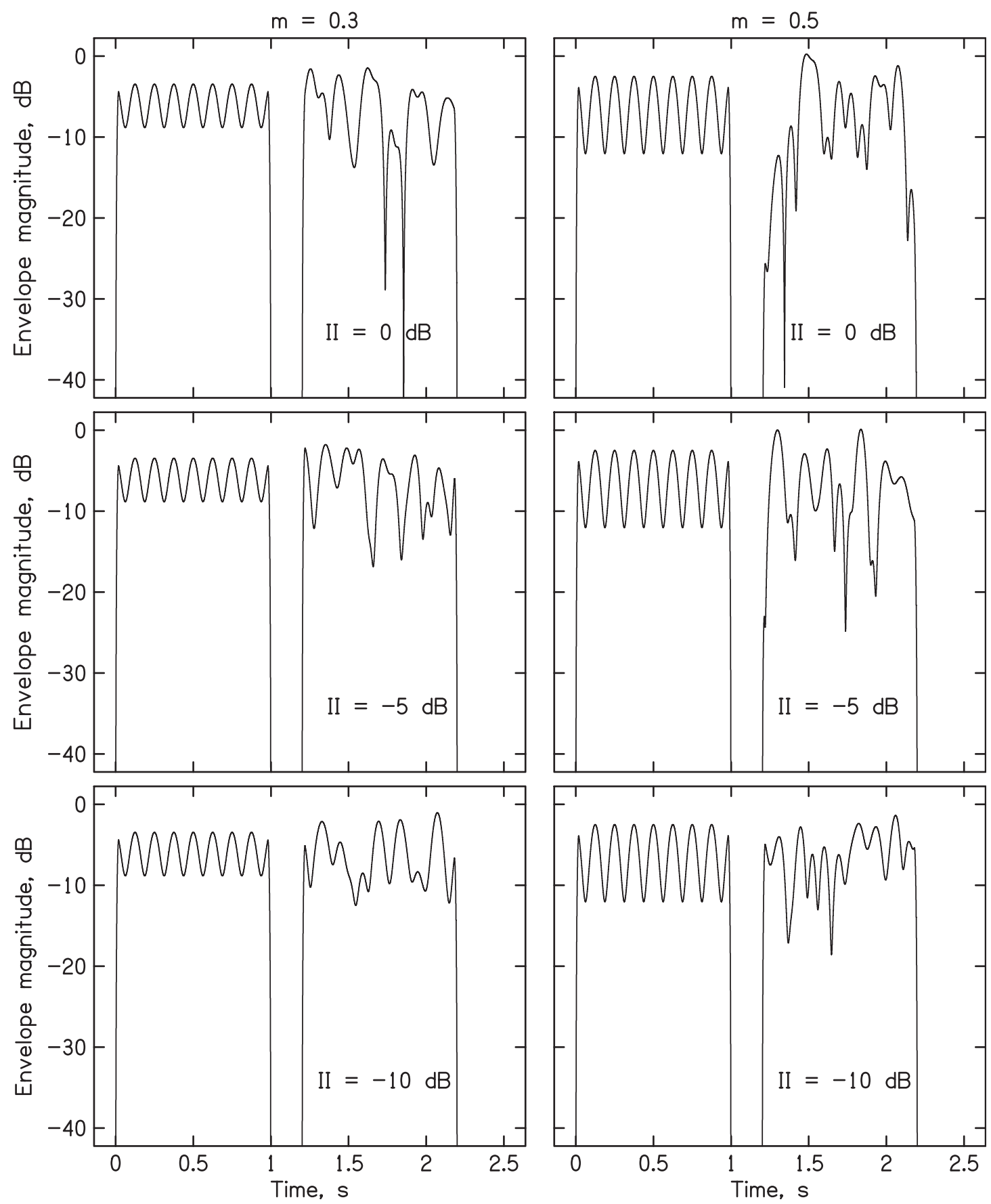

FIG. 1. Examples of the envelopes of stimuli with $m=0.3$ (left) and $m=0.5$ (right) for three values of the II, $0,-5$, and $-10 \mathrm{~dB}$ (rows). The signal interval is the second for all examples. The envelopes are plotted on a dB scale. A fully modulated carrier with no irregularity $(m=1)$ would have a peak envelope magnitude of $0 \mathrm{~dB}$. 
intervals). For a fixed value of the II, the ratio $\mathrm{PVR}_{\mathrm{SD}} /$ $\mathrm{PVR}_{\text {mean }}$ is approximately constant across different values of $m$ for $m$ in the range $0.2-0.5$. This leads to the prediction that the threshold II should be invariant with $m$ over this range.

In a case of hearing loss with loudness recruitment, the effect of the hearing loss is similar to that of magnification of the PVR by a certain factor, when the modulation depth is expressed in dB (Moore et al., 1996). Such magnification would not affect the ratio $\mathrm{PVR}_{\mathrm{SD}} / \mathrm{PVR}_{\text {mean }}$. Hence, if the data support the prediction that the threshold II is invariant with $m$, this suggests that loudness recruitment would not affect the outcome of the ERD test.

The starting value of the II was usually $9.5 \mathrm{~dB}$, a large value, so as to make it easier to "know what to listen for." The value of the II was varied from trial to trial using an adaptive two-down one-up procedure to estimate the value of the II leading to $70.7 \%$ correct. The II was changed by a factor of 1.33 (in linear units) until two reversals had occurred and by a factor of 1.1 until six more reversals occurred. To avoid "over-modulation" the maximum value of the II needs to be limited. In practice, we found that setting the maximum value of the II to $9.5 \mathrm{~dB}$ worked well for values of $m$ up to 0.5 , this limit only occasionally being reached during an adaptive run. Hence, that limit was used in the experiments reported here. Whenever the adaptive procedure called for an II greater than $9.5 \mathrm{~dB}$, the II was set to $9.5 \mathrm{~dB}$. The threshold was estimated as the geometric mean of the values of the II at the last six reversal points. Thresholds that are close to $9 \mathrm{~dB}$ might result from random guessing, reflecting either a complete inability to perform the task or a failure to understand "what to listen for."

Synaptopathy may selectively affect auditory neurons with low spontaneous rates and high thresholds (Furman et al., 2013). To reveal such an effect, the ERD test can be conducted using a relatively high signal level, for example, $80 \mathrm{~dB}$ sound pressure level (SPL), in the presence of a broadband background noise to limit the range of characteristic frequencies of the neurons that respond to the signal. In the present study, the noise started $200 \mathrm{~ms}$ before the onset of the first stimulus in a trial and finished $200 \mathrm{~ms}$ after the second stimulus. The noise used is called "threshold equalizing noise" (TEN; Moore et al., 2000). TEN is designed to produce equal masked thresholds for sinusoidal signals over a wide frequency range. The TEN level is specified as the level in a $1-\mathrm{ERB}_{\mathrm{N}}$-wide band centered at $1000 \mathrm{~Hz}$, where $\mathrm{ERB}_{\mathrm{N}}$ stands for the mean value of the equivalent rectangular bandwidth of the auditory filter for people with normal hearing (Glasberg and Moore, 1990). In the present study, the TEN level was set $25 \mathrm{~dB}$ below the level of the signal in all experiments.

Although animal studies suggest that noise exposure selectively affects high-threshold neurons, there is evidence that it can also affect the perception of low-level sounds (Stone et al., 2008; Stone and Moore, 2014). Hence, we also evaluated performance on the ERD test using stimuli presented at $20 \mathrm{~dB}$ sensation level (SL).

All testing in the present study was conducted in quiet rooms, usually double-walled sound-attenuating rooms.
Stimuli were generated using personal computers (PCs) with a sample rate of $48000 \mathrm{~Hz}$ and 24-bit precision. Stimuli were converted to analog form using sound cards with 24-bit precision and presented to one ear via Sennheiser HDA200 headphones (Wedemark, Germany). Different subjects were used for each experiment.

Audiometric thresholds were assessed using an Interacoustics AC40 audiometer (Middlefart, Germany) and Sennheiser HDA300 headphones (Wedemark, Germany) in experiments 1 and 2, an Otometrics (Taastrup, Denmark) Aurical Otosuite or Primus and Telephonics (Huntington, NY) TDH-39 P headphones in experiment 3, and a Kamplex (London, UK) KS8 audiometer with TDH-39 headphones in experiment 4. The method recommended by the British Society of Audiology (2011) was used to assess audiometric thresholds.

\section{EXPERIMENT I: ASSESSMENT OF LEVEL EFFECTS AND PRACTICE}

Tests intended for use in large-scale research studies or the clinic should require minimal practice to achieve stable results. Experiment 1 was conducted to assess how much practice was needed to obtain stable results for the ERD test. A second aim was to assess the effect of stimulus level on performance of the ERD test. A third aim was to assess how the results for the ERD test were related to lifetime noise exposure, as estimated using the Noise Exposure Structured Interview (NESI; Guest et al., 2018a). The measure obtained using the NESI is intended to be linearly related to the total energy of exposure above $80 \mathrm{dBA}$ (Guest et al., 2018a). One unit is equivalent to one working year $(2080 \mathrm{~h})$ of exposure to $90 \mathrm{dBA}$.

\section{A. Subjects}

Twenty subjects were tested, half of whom were female. Their ages ranged from 20 to 41 years [mean $=27$ years, standard deviation $(\mathrm{SD})=5 \mathrm{~dB}]$. Each subject was tested using the right ear only. For the test ear, all subjects had audiometric thresholds better than or equal to $20 \mathrm{~dB}$ hearing level (HL) for frequencies from 250 to $8000 \mathrm{~Hz}$ except for subject $S_{6}$ who had a threshold of $25 \mathrm{~dB} \mathrm{HL}$ at $8 \mathrm{kHz}$ and subject $S_{17}$ who had a threshold of $25 \mathrm{~dB} \mathrm{HL}$ at $500 \mathrm{~Hz}$. Audiometric thresholds at the test frequency of $4000 \mathrm{~Hz}$ ranged from -5 to $15 \mathrm{~dB} \mathrm{HL}$ with a mean of $3.8 \mathrm{~dB} \mathrm{HL}$. The cumulative noise exposure of each participant was estimated using the NESI (Guest et al., 2018a). Unfortunately, due to time constraints, it was not possible to administer the NESI for two subjects, $S_{8}$ and $S_{9}$.

\section{B. Stimuli and conditions}

Each subject was tested using two levels of the $4000-\mathrm{Hz}$ sinusoidal carrier, $80 \mathrm{~dB}$ SPL and $20 \mathrm{~dB}$ SL. To set the SL, absolute thresholds for a $4000-\mathrm{Hz} 1000-\mathrm{ms}$ sinusoid were determined using a $2 \mathrm{AFC}$ task and a two-down, one-up procedure tracking the $70.7 \%$ correct point on the psychometric function. The absolute threshold was estimated twice and the mean of the two estimates was used. For the ERD test, the 
modulation index of the non-signal stimulus was 0.3 and $f_{m}$ was $8 \mathrm{~Hz}$.

Subjects $S_{1}-S_{10}$ were tested first using nine runs at $80 \mathrm{~dB}$ SPL. These were divided into three blocks of three with a brief break in between. They were then tested using one block of three runs at $20 \mathrm{~dB}$ SL. Subjects $S_{11}-S_{20}$ were tested first using nine runs at $20 \mathrm{~dB}$ SL, again divided into three blocks of three with a brief break in between. They were then tested using a block of three runs at $80 \mathrm{~dB}$ SPL.

\section{Results}

The thin lines with symbols in Fig. 2 show the individual results. The thick grey lines show the means. There was substantial individual variability. However, for the thresholds averaged across blocks of three runs (lower panels), all thresholds were below $5 \mathrm{~dB}$, indicating that all subjects were able to perform the task. The results within each subject were reasonably consistent. Thresholds tended to decrease slightly across the first 2-3 runs and then to remain stable. To compare results for the two levels, mean thresholds were calculated ignoring the thresholds for the first two runs for each subject. There was no significant difference in mean thresholds for the two levels; mean thresholds were -5.1 and $-4.8 \mathrm{~dB}$ at $80 \mathrm{~dB}$ SPL and $20 \mathrm{~dB}$ SL, respectively. Individual variability was somewhat larger at $80 \mathrm{~dB}$ SPL than at $20 \mathrm{~dB}$ SL; the SD across subjects was $4.3 \mathrm{~dB}$ at $80 \mathrm{~dB}$ SPL and $2.8 \mathrm{~dB}$ at $20 \mathrm{~dB}$ SL. Based on a variance-ratio $(F)$ test, the difference in SDs was significant $[F(19,19)=2.35$, $p<0.05]$. There was a significant high correlation between thresholds for the two levels: $r=0.86, p<0.001$. This shows that performance differences between subjects were consistent across levels. The ERD thresholds were not significantly correlated with the audiometric thresholds at $4000 \mathrm{~Hz}$ for either level ( $r=0.17$ at $80 \mathrm{~dB}$ SPL and $r=0.26$ at $20 \mathrm{~dB}$ SL, both $p>0.05)$. Variations in absolute threshold probably partly reflect variations in the functioning of the active mechanism mediated by the OHCs, so the lack of correlation is consistent with the idea that performance of the ERD test is not affected by small variations in the functioning of the $\mathrm{OHCs}$.

For the 18 subjects for whom the NESI was administered, scores ranged from 4 to 268 units, where higher numbers indicate more exposure. NESI scores were not significantly correlated with ERD thresholds obtained at $20 \mathrm{~dB}$ SL $(r=-0.34, p=0.17)$. NESI scores were significantly negatively correlated with ERD thresholds obtained at $80 \mathrm{~dB}$ SPL $(r=-0.62, p<0.01)$, the opposite of what would be expected if ERD thresholds increased with increasing noise-induced synaptopathy. However, there is a likely confounding factor in that the subjects with the highest NESI scores tended to be musicians or, in one case, a keen audiophile. Such people may have high "processing efficiency," i.e., a better-than-average ability to use the available neural information. In other words, they may be "good listeners." This could account for why ERD thresholds obtained at $80 \mathrm{~dB}$ SPL were negatively correlated with NESI scores.

The individual variability in the ERD thresholds may reflect individual differences in HHD, or differences in processing efficiency, or a combination of the two. A possible explanation for the lower individual variability at $20 \mathrm{~dB}$ SL than at $80 \mathrm{~dB}$ SPL is as follows. As described in the Introduction, synaptopathy may mainly affect neurons with high thresholds (Furman et al., 2013). Hence, the results obtained at $20 \mathrm{~dB}$ SL may be largely unaffected by synaptopathy, and individual differences may mainly reflect differences
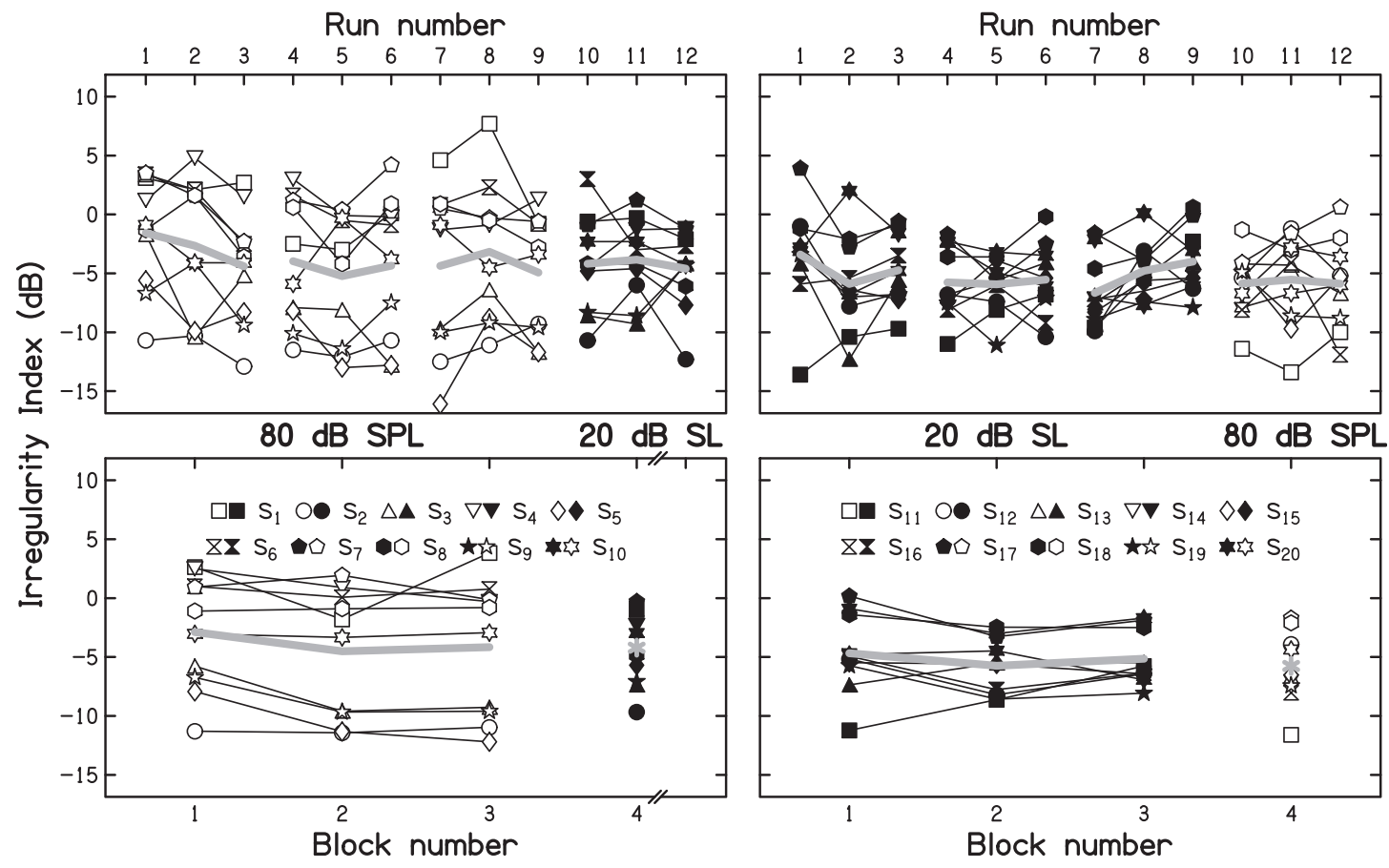

FIG. 2. Individual results (symbols connected by thin lines) and mean results (thick gray lines) for experiment 1 . Thresholds obtained at $80 \mathrm{~dB}$ SPL and $20 \mathrm{~dB}$ SL are shown by open and closed symbols, respectively. The panels at the top show thresholds for each run and the panels at the bottom show the average thresholds for each block of three runs. Thresholds for subjects $S_{1}-S_{10}$ are shown on the left and thresholds for subjects $S_{11}-S_{20}$ are shown on the right. 
in processing efficiency. On the other hand, individual variability in the ERD thresholds obtained at $80 \mathrm{~dB}$ SPL may reflect both differences in processing efficiency and differences in the degree of synaptopathy. If this reasoning is correct, then the difference between ERD thresholds obtained at $80 \mathrm{~dB}$ SPL and at $20 \mathrm{~dB}$ SL (80-20) might provide a measure of the effect of synaptopathy that is not greatly influenced by individual differences in processing efficiency. However, the results obtained using the NESI are not consistent with this reasoning. The differences in ERD thresholds were negatively rather than positively correlated with the NESI scores $(r=-0.72$, $p<0.001)$. One way to account for this result is to assume that synaptopathy has greater effects for low-level sounds than for high-level sounds. However, this would not explain why individual variability was lower at $20 \mathrm{~dB}$ SL than at $80 \mathrm{~dB}$ SPL.

In summary, all subjects could perform the task, but there was substantial individual variability. Variability was smaller at $20 \mathrm{~dB}$ SL than at $80 \mathrm{~dB}$ SPL. Thresholds were mostly consistent within each subject and across levels.

\section{EXPERIMENT 2: EFFECTS OF CARRIER FREQUENCY AND EAR OF PRESENTATION}

The test frequency of $4000 \mathrm{~Hz}$ used in experiment 1 was chosen since noise-induced hearing loss is typically greatest for that frequency (Borg et al., 1995). This probably happens because of two factors. First the ear-canal resonance leads to an increase in sound level relative to that in free field for frequencies close to $3000 \mathrm{~Hz}$ (Shaw, 1974). Second, leveldependent shifts in the position of peak vibration on the basilar membrane have the effect that at high levels a $3000-\mathrm{Hz}$ tone produces maximum vibration at the place that (at levels close to threshold) is tuned to $4000 \mathrm{~Hz}$ (McFadden, 1986; Robles and Ruggero, 2001; Moore et al., 2002). Hence, when exposed to intense broadband stimuli, the highest basilar membrane vibration and the greatest damage occur at a place that is usually tuned to about $4000 \mathrm{~Hz}$. It seems plausible that HHD is greatest for frequencies where the effective exposure level is maximal, i.e., frequencies close to $4000 \mathrm{~Hz}$. If the individual differences found in experiment 1 reflect differences in $\mathrm{HHD}$, and if, as seems likely, HDD varies across frequency, then thresholds on the ERD test should correspondingly vary across frequency (and perhaps across ears).

Another possibility is that the individual differences found in experiment 1 reflect differences in processing efficiency. If so, thresholds on the ERD test should be similar across frequency (and across ears). These two possibilities were assessed in experiment 2.

\section{A. Subjects}

Twelve subjects were tested, eight of whom were female. Their ages ranged from 21 to 27 years (mean$=24$ years, $\mathrm{SD}=2$ years). All had audiometric thresholds of $15 \mathrm{~dB}$ HL or better for all frequencies from 250 to $8000 \mathrm{~Hz}$ in both ears.

\section{B. Stimuli and conditions}

As for experiment 1 , the modulation index of the nonsignal stimulus was 0.3 and $f_{m}$ was $8 \mathrm{~Hz}$. The signal level was $80 \mathrm{~dB}$ SPL. Each subject was tested using one ear with $f_{c}=1000,2000$, and $4000 \mathrm{~Hz}$ and the other ear with $f_{c}=4000 \mathrm{~Hz}$, giving four conditions. Six randomly chosen subjects were tested with the three different carriers presented to the right ear and the remaining six were tested with the three different carriers presented to the left ear. The order of testing the four conditions was counterbalanced across subjects. Two practice runs were given for the condition that was tested first. Then, three runs were given for each condition.

\section{Results}

The mean thresholds across the three runs for each condition are shown in Fig. 3. For most subjects, thresholds were reasonably constant across frequencies and ears. In the few cases where there were large across-frequency variations (e.g., $S_{11}$, asterisks), the SDs across the three thresholds for a given condition were relatively large, so the acrossfrequency variations might just reflect random variability in the measurements or lapses of attention. One subject $\left(S_{5}\right.$, diamonds) had thresholds close to $9 \mathrm{~dB}$, indicating nearchance performance.

A within-subjects analysis of variance (ANOVA) was conducted with factor $f_{c}$, excluding the data for $S_{5}$. Mauchley's test for sphericity was significant, so the Greenhouse-Geisser correction was used, but the uncorrected degrees of freedom are reported. There was no significant effect of $f_{c} ; F(2,20)=2.97, p=0.092$. Thresholds at $4000 \mathrm{~Hz}$ were correlated across the two ears $(r=0.87, p<0.01)$. Thresholds within one ear were correlated across all pairs of frequencies: 1000 versus $2000 \mathrm{~Hz}, r=0.67, p=0.024 ; 2000$ versus $4000 \mathrm{~Hz}, r=0.88, p<0.001 ; 1000$ versus $4000 \mathrm{~Hz}$, $r=0.77, p<0.01)$. Overall, these outcomes are broadly consistent with the idea that the individual differences result

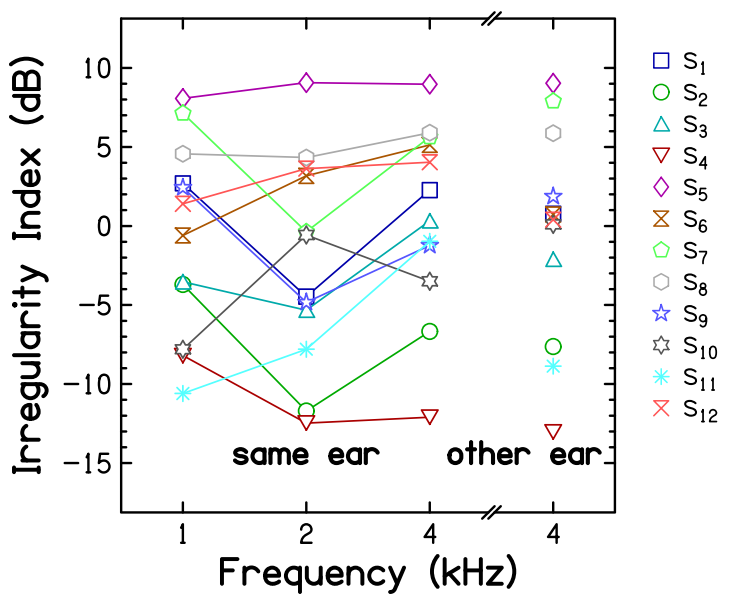

FIG. 3. (Color online) Results of experiment 2, showing individual thresholds averaged across three runs for each condition. Each subject is represented by a different symbol. The three leftmost points show thresholds for the three carrier frequencies that were tested using one ear. The rightmost points show the threshold for the $4000-\mathrm{Hz}$ carrier when presented to the other ear. 
largely from differences in processing efficiency. However, it remains possible that the poorly performing subjects had some degree of HDD, and that this affected thresholds roughly equally for all carrier frequencies and both ears.

\section{EXPERIMENT 3: EFFECT OF MODULATION DEPTH}

This experiment explored the effect of varying the baseline modulation depth, $m$. It was hoped that the thresholds for the ERD test would be almost independent of the value of $m$. If so, this would support the use of the test with hearing-impaired people. Such people often experience loudness recruitment, which increases the perceived amount of modulation (Moore et al., 1996). As noted earlier, this effect is similar to that of increasing the amount of modulation (the PVR in $\mathrm{dB}$ ) by a certain factor for people with normal hearing. If performance of the ERD test depends on the amount of irregularity relative to the baseline modulation index, i.e., on $\mathrm{PVR}_{\mathrm{SD}} / \mathrm{PVR}_{\text {mean }}$, then thresholds for the ERD test should be largely independent of $m$. If so, this would mean that loudness recruitment should have little effect on the outcome of the ERD test.

We wanted to use values of $m$ that resulted in clearly audible modulation in the non-signal interval, while avoiding frequent approaches to the limit of $\mathrm{II}=9.5 \mathrm{~dB}$ (which was imposed to prevent over-modulation) in the signal interval. Pilot experiments showed that values of $m$ in the range $0.2-0.5$ satisfied these criteria. Hence, the experiment was conducted using $m=0.2,0.3,0.4$, and 0.5 .

\section{A. Subjects}

Sixteen subjects were tested, ten of whom were female. Their ages ranged from 20 to 28 years (mean $=23$ years, $\mathrm{SD}=2$ years). Half of the subjects were tested using the right ear and half using the left ear. For the test ear, all subjects had audiometric thresholds better than or equal to $20 \mathrm{~dB}$ HL for frequencies from 250 to $8000 \mathrm{~Hz}$.

\section{B. Stimuli and conditions}

The value of $f_{m}$ was $8 \mathrm{~Hz}$. The values of $m$ were $0.2,0.3$, 0.4 , and 0.5 . The level was $80 \mathrm{~dB}$ SPL. At least two practice runs with $m=0.3$ were given before testing proper started. The order of testing the different values of $m$ was counterbalanced across subjects. Four threshold runs were obtained for each value of $m$ before moving on to the next value of $m$. The final threshold for each value of $m$ was estimated as the mean for the four runs.

\section{Results}

Figure 4 shows the threshold for each subject as a function of $m$. As for experiments 1 and 2 , individual variability was large. One subject (up-pointing arrows) had thresholds close to $9 \mathrm{~dB}$, indicating near-chance performance, for all values of $m$. The mean thresholds across subjects, excluding the data for the subject who performed near chance, were -0.2 , $-1.2,-2.1$, and $-2.0 \mathrm{~dB}$ for $m=0.2,0.3,0.4$, and 0.5 , respectively. A within-subjects ANOVA with factor $m$, again excluding the data for the subject who performed near chance,

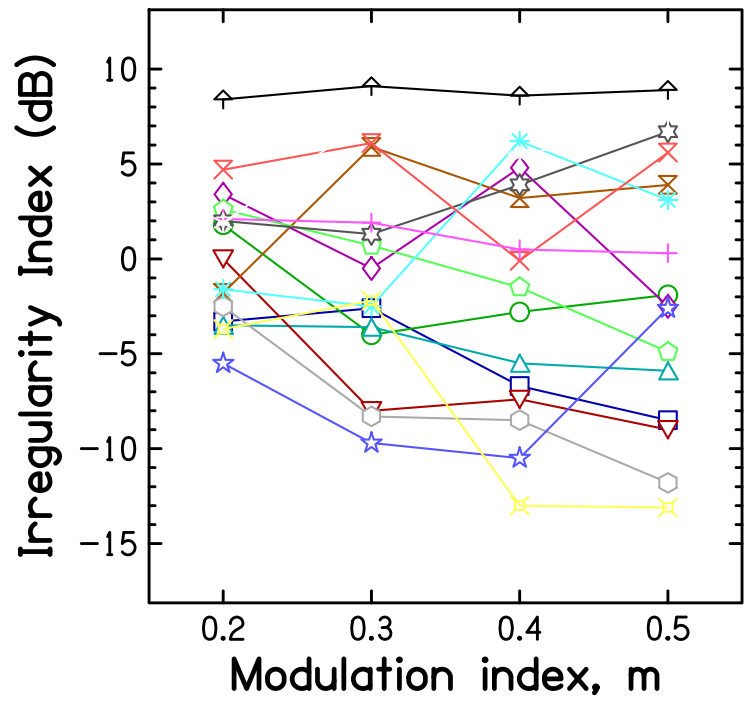

FIG. 4. (Color online) Results of experiment 3, showing the threshold for each subject as a function of $m$.

showed no significant effect; $F(3,56)=0.39, p=0.76$. This result indicates that performance on the ERD test is almost independent of $m$ and implies that the outcome of the ERD test should be little affected by the presence of loudness recruitment, since recruitment would have the effect of magnifying the effective "internal" modulation depth by the same factor in the two intervals (Moore et al., 1996).

\section{EXPERIMENT 4: EFFECT OF MODULATION RATE}

Experiments 1-3 were all conducted using $f_{m}=8 \mathrm{~Hz}$. This rate was chosen so that several modulation cycles would occur within the 1000-ms duration of each stimulus, while at the same time the amplitude fluctuations could be easily "followed." Experiment 4 was conducted to assess how performance on the ERD test varied with $f_{m}$ and whether individual variability changed with $f_{m}$. Decreasing $f_{m}$ leads to a reduced number of modulation cycles within the stimulus duration, which might make performance worse (Sheft and Yost, 1990), but this effect might be offset by an improved ability to "follow" the slower amplitude fluctuations in the stimuli. Experiment 4 explored how the balance between these two factors affected performance of the ERD test.

\section{A. Subjects}

Eight subjects were tested, four of whom were female. Their ages ranged from 18 to 21 years (mean $=19$ years, $\mathrm{SD}=1.5$ years). Half of the subjects were tested using the right ear and half using the left ear. All had audiometric thresholds of $20 \mathrm{~dB}$ HL or better for all frequencies from 250 to $8000 \mathrm{~Hz}$ for the test ear, except for $S_{7}$, who had an audiometric threshold of $30 \mathrm{~dB} \mathrm{HL}$ at $250 \mathrm{~Hz}$.

\section{B. Stimuli and conditions}

The carrier frequency was $4000 \mathrm{~Hz}$, the carrier level was $80 \mathrm{~dB}$ SPL, and the value of $m$ was 0.3 . Values of $f_{m}$ were 2, 4,8 , and $16 \mathrm{~Hz}$. At least two practice runs with $f_{m}=8 \mathrm{~Hz}$ 
were given before testing proper started. The order of testing the different values of $f_{m}$ was counter-balanced across subjects. Four threshold runs were obtained for each value of $f_{m}$ before moving on to the next value of $f_{m}$. The final threshold for each value of $f_{m}$ was estimated as the mean for the four runs.

\section{Results}

Figure 5 shows the individual thresholds (open symbols and thin lines). One subject $\left(S_{8}\right)$ had thresholds close to $9 \mathrm{~dB}$, indicating near-chance performance. The mean thresholds across subjects (thick line connected by filled circles) were calculated excluding the results for $S_{8}$. A withinsubjects ANOVA was conducted with factor $f_{m}$, excluding the data for $S_{8}$. Mauchley's test for sphericity was significant, so the Greenhouse-Geisser correction was used, but the uncorrected degrees of freedom are reported. The effect of $f_{m}$ was significant: $F(3,21)=6.02, p=0.025$, thresholds tending to decrease with increasing $f_{m}$. Pairwise post hoc comparisons, based on Fisher's protected least-significantdifference (LSD) test, showed that thresholds differed significantly $(p<0.05)$ for the following pairs of values of $f_{m}: 2$ and $4 \mathrm{~Hz}, 2$ and $8 \mathrm{~Hz}, 2$ and $16 \mathrm{~Hz}$. However, there was considerable individual variability in the pattern of results across $f_{m}$. For example, $S_{3}$ showed a very large decrease in threshold with increasing $f_{m}$, while $S_{2}$ showed a moderate increase.

The SD across subjects also tended to increase with increasing $f_{m}$. Excluding the results for $S_{8}$, the SD was 2.2, $2.7,4.2$, and $6.5 \mathrm{~dB}$ for the rates of $2,4,8$, and $16 \mathrm{~Hz}$, respectively. Based on variance ratio $(F)$ tests, individual variability was significantly greater at 16 than at $2 \mathrm{~Hz}[F(8,8)=8.73$, $p<0.01]$ and at 16 than at $4 \mathrm{~Hz}[F(8,8)=5.80, p<0.02]$.

\section{DISCUSSION}

Across all four experiments there was substantial individual variability. The results of experiment 2 showed that the thresholds for each subject usually varied only slightly across carrier frequencies and across ears for $f_{c}=4000 \mathrm{~Hz}$.

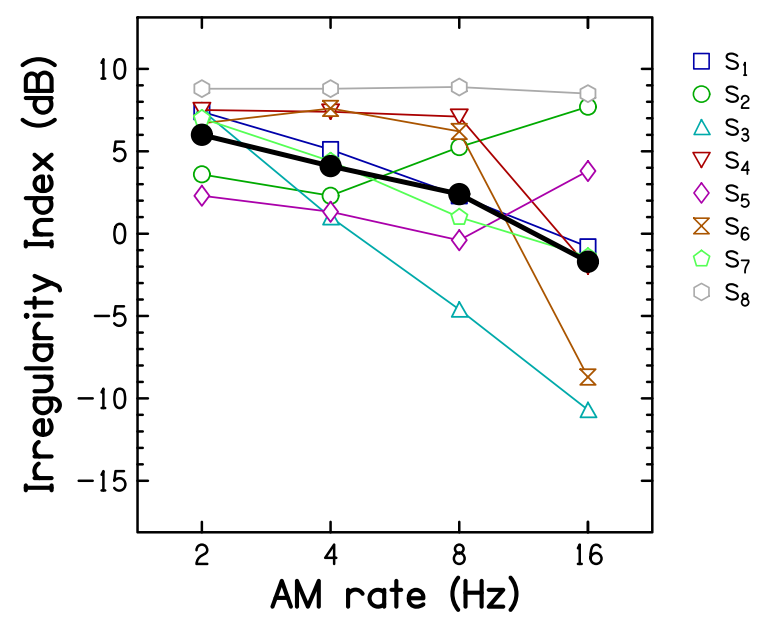

FIG. 5. (Color online) Results of experiment 4, showing individual thresholds averaged across the four runs for each value of $f_{m}$. Each subject is represented by a different symbol. The thick line connecting filled circles shows the mean across subjects, excluding $S_{8}$, who performed close to chance.
Also, in experiment 1 , there was a high correlation between thresholds obtained at $80 \mathrm{~dB}$ SPL and $20 \mathrm{~dB}$ SL. These findings indicate a good degree of consistency within subjects but poor consistency across subjects, broadly supporting the idea that the individual variability reflects differences across subjects in processing efficiency rather than differences in the quality of the neural encoding of the envelopes of the stimuli. It is possible that the poorly performing subjects had some form of HHD that was fairly uniform across frequencies and across ears, but this seems unlikely given that all of the subjects were relatively young and had audiometric thresholds within the normal range at the test frequency. One subject in each of experiments 2, 3, and 4 gave thresholds that were close to $9 \mathrm{~dB}$ for all conditions, indicating nearchance performance. It is possible that these three subjects had very poor neural coding of the envelopes of the stimuli, but it seems more likely that they simply failed to grasp what to listen for.

Mean thresholds for the ERD test varied across experiments from about $-4 \mathrm{~dB}$ (experiment 1) to $1 \mathrm{~dB}$ (experiment 2 ). These mean thresholds correspond roughly to the highest amount of irregularity illustrated in Fig. $1(\mathrm{II}=0 \mathrm{~dB})$. This indicates that a typical subject needs a rather high degree of envelope irregularity for that irregularity to be detected. However, some subjects (e.g., subject $S_{2}$ in experiment 1) consistently achieved thresholds close to $-10 \mathrm{~dB}$, the value illustrated by the smallest amount of irregularity in Fig. 1.

The results of experiment 1 showed that thresholds typically reached asymptotic values after two threshold runs. However, even after several runs had been performed, some subjects showed erratic performance, with thresholds close to the II of $9 \mathrm{~dB}$ indicating near-chance performance. For example, one subject in experiment 3 (picnic-table symbols in Fig. 4), who had already completed two practice runs with $m=0.3$ and four test runs with $m=0.2$, gave thresholds of 8.6 and $8.7 \mathrm{~dB}$ when first tested with $m=0.4$, but then gave thresholds of -8.1 and $-9.4 \mathrm{~dB}$. The high thresholds may reflect loss of concentration or a failure to attend to the appropriate detection cues.

The results of experiment 3 showed that mean thresholds did not vary significantly with $m$ over the range $0.2-0.5$. This indicates that performance of the ERD test does not depend strongly on the absolute amount of AM, provided that the AM in the non-signal interval is clearly audible. Rather, performance appears to depend on the amount of irregularity in the AM relative to the baseline amount of AM, a behavior consistent with Weber's law. This finding suggests that the results of the ERD test should be almost unaffected by the presence of loudness recruitment, since recruitment should magnify the internal representation of both the baseline AM and the irregularity in the AM (Moore et al., 1996). This is in contrast with AM detection, for which loudness recruitment can lead to improved performance for stimuli presented at low SLs (Jerger, 1962; Ernst and Moore, 2012; Schlittenlacher and Moore, 2016).

The results of experiment 4 showed that mean thresholds for the ERD test decreased with increasing $f_{m}$, although not all subjects showed this effect. The improvement in performance with increasing $f_{m}$ may reflect a beneficial effect of 
having an increased number of AM cycles within the fixed stimulus duration (Sheft and Yost, 1990). The more AM cycles there are, the greater the chances of a distinct irregularity occurring, for example, an unusually large peak in the envelope. However, the improvement in mean threshold with increasing $f_{m}$ was accompanied by an increase in individual variability.

As described in the Introduction, the ERD test was developed partly with the aim of using it as a method of diagnosing HHD. The large individual variability found across all four experiments limits the usefulness of the ERD test for this purpose. However, the ERD test may still be useful for detecting the presence of across-frequency variations in HHD, for example, in populations that have been exposed to high levels of noise. The ERD test may also be useful for comparing the fidelity of envelope coding before and after noise exposure, for example, among military personnel or among those who attend concerts with very high sound levels. For a test to be used in this way, the test duration should be reasonably short. On average, a single run of the ERD test took about $3 \mathrm{~min}$. After an initial practice run, the standard deviation across repeated runs for a given subject was typically about $2 \mathrm{~dB}$. Hence, to get an estimate of threshold within $2 \mathrm{~dB}$ of the "true" mean would typically require at least one practice run and 2-3 test runs, taking about $10-13 \mathrm{~min}$ in total. This seems reasonable for use in largescale studies.

\section{CONCLUSIONS}

A new test, called the ERD test, was developed to assess the ability to discriminate irregular from regular AM. The amount of irregularity was parametrically varied and quantified by the II, expressed in $\mathrm{dB}$. It was hoped that the test might be useful for detecting HHD. Normative data were gathered for young subjects with normal audiometric thresholds. All tests were performed using a background TEN to limit the range of center frequencies conveying useful information. The main findings were the following:

(1) Performance of the ERD test using $f_{c}=4000 \mathrm{~Hz}$, $f_{m}=8 \mathrm{~Hz}$, and $m=0.3$ improved over the first two threshold runs and then remained roughly stable, except for occasional lapses.

(2) There was a high correlation between thresholds obtained at $80 \mathrm{~dB}$ SPL and $20 \mathrm{~dB}$ SL using $f_{c}=4000 \mathrm{~Hz}$, $f_{m}=8 \mathrm{~Hz}$, and $m=0.3$.

(3) Thresholds obtained using $f_{m}=8 \mathrm{~Hz}$ and $m=0.3$ with a level of $80 \mathrm{~dB}$ SPL did not vary significantly across $f_{c}=1000,2000$, and $4000 \mathrm{~Hz}$, and thresholds obtained with $f_{c}=4000 \mathrm{~Hz}$ did not vary significantly across ears.

(4) Thresholds obtained using $f_{m}=8 \mathrm{~Hz}$ and $f_{c}=4000 \mathrm{~Hz}$ with a level of $80 \mathrm{~dB}$ SPL did not vary significantly with $m$ for $m=0.2-0.5$.

(5) Thresholds obtained using $m=0.3$ and $f_{c}=4000 \mathrm{~Hz}$ with a level of $80 \mathrm{~dB}$ SPL tended to improve with increasing $f_{m}$ from 2 to $16 \mathrm{~Hz}$. However, the individual variability also increased with increasing $f_{m}$.

(6) The large individual differences among young normalhearing subjects probably largely reflect differences in processing efficiency rather than the fidelity of the neural coding of AM, limiting the usefulness of the ERD test for detecting HHD. Nevertheless, the ERD test may still be useful for detecting across-frequency variations in HHD and comparing the fidelity of envelope coding before and after noise exposure.

\section{ACKNOWLEDGMENTS}

This work was supported by the Engineering and Physical Sciences Research Council (UK, Grant No. RG78536). We thank Brian Glasberg for help with statistical analyses and Rosie Cave for help with data collection. We also thank two reviewers for helpful comments on an earlier version of this paper.

Bacon, S. P., and Gleitman, R. M. (1992). "Modulation detection in subjects with relatively flat hearing losses," J. Speech Hear. Res. 35, 642-653.

Bacon, S. P., and Viemeister, N. F. (1985). "Temporal modulation transfer functions in normal-hearing and hearing-impaired subjects," Audiology 24, 117-134.

Bharadwaj, H. M., Masud, S., Mehraei, G., Verhulst, S., and ShinnCunningham, B. G. (2015). "Individual differences reveal correlates of hidden hearing deficits," J. Neurosci. 35, 2161-2172.

Borg, E., Canlon, B., and Engström, B. (1995). "Noise-induced hearing loss-Literature review and experiments in rabbits. Morphological and electrophysiological features, exposure parameters and temporal factors, variability and interactions," Scand. Audiol. 24, Suppl. 40, 1-147.

Bramhall, N. F., Konrad-Martin, D., McMillan, G. P., and Griest, S. E. (2017). "Auditory brainstem response altered in humans with noise exposure despite normal outer hair cell function," Ear Hear. 38, e1-e12.

British Society of Audiology (2011). Pure-Tone Air-Conduction and BoneConduction Threshold Audiometry with and without Masking: Recommended Procedure (British Society of Audiology, Reading, UK).

Burns, E. M., and Viemeister, N. F. (1976). "Nonspectral pitch," J. Acoust. Soc. Am. 60, 863-869.

Dobie, R. A., and Humes, L. E. (2017). "Commentary on the regulatory implications of noise-induced cochlear neuropathy," Int. J. Audiol. 56, 74-78.

Ernst, S. M., and Moore, B. C. J. (2012). "The role of time and place cues in the detection of frequency modulation by hearing-impaired listeners," J. Acoust. Soc. Am. 131, 4722-4731.

Füllgrabe, C., Meyer, B., and Lorenzi, C. (2003). "Effect of cochlear damage on the detection of complex temporal envelopes," Hear. Res. 178, $35-43$.

Füllgrabe, C., Moore, B. C. J., and Stone, M. A. (2015). “Age-group differences in speech identification despite matched audiometrically normal hearing: Contributions from auditory temporal processing and cognition," Front. Aging Neurosci. 6, 347, 1-25.

Furman, A. C., Kujawa, S. G., and Liberman, M. C. (2013). "Noise-induced cochlear neuropathy is selective for fibers with low spontaneous rates," J. Neurophysiol. 110, 577-586.

Glasberg, B. R., and Moore, B. C. J. (1990). "Derivation of auditory filter shapes from notched-noise data," Hear. Res. 47, 103-138.

Grose, J. H., Buss, E., and Hall, J. W., III (2017). "Loud music exposure and cochlear synaptopathy in young adults: Isolated auditory brainstem response effects but no perceptual consequences," Trends Hear. 21, 1-18.

Guest, H., Dewey, R. S., Plack, C. J., Couth, S., Prendergast, G., Bakay, W., and Hall, D. A. (2018a). "The noise exposure structured interview (NESI): An instrument for the comprehensive estimation of lifetime noise exposure," Trends Hear. 22, 1-20.

Guest, H., Munro, K. J., Prendergast, G., Millman, R. E., and Plack, C. J. (2018b). "Impaired speech perception in noise with a normal audiogram: No evidence for cochlear synaptopathy and no relation to lifetime noise exposure," Hear. Res. 364, 142-151.

Jerger, J. (1962). “The SISI test,” Int. Audiol. 1, 246-247.

Kujawa, S. G., and Liberman, M. C. (2009). "Adding insult to injury: Cochlear nerve degeneration after 'temporary' noise-induced hearing loss," J. Neurosci. 29, 14077-14085. 
Kujawa, S. G., and Liberman, M. C. (2015). "Synaptopathy in the noiseexposed and aging cochlea: Primary neural degeneration in acquired sensorineural hearing loss," Hear. Res. 330, 191-199.

Lemanska, J., Skrodzka, E., and Sek, A. (2002). "Discrimination of the amplitude modulation rate," Arch. Acoust. 27, 3-22.

Liberman, M. C., Epstein, M. J., Cleveland, S. S., Wang, H., and Maison, S. F. (2016). "Toward a differential diagnosis of hidden hearing loss in humans," PLoS One 11, e0162726.

Liberman, M. C., and Kujawa, S. G. (2017). "Cochlear synaptopathy in acquired sensorineural hearing loss: Manifestations and mechanisms," Hear. Res. 349, 138-147.

McFadden, D. (1986). "The curious half octave shift: Evidence for a basalward migration of the travelling-wave envelope with increasing intensity," in Basic and Applied Aspects of Noise-Induced Hearing Loss, edited by R. J. Salvi, D. Henderson, R. P. Hamernik, and V. Colletti (Plenum, New York), pp. 295-312.

Moore, B. C. J. (2014). Auditory Processing of Temporal Fine Structure: Effects of Age and Hearing Loss (World Scientific, Singapore), pp. 1-182.

Moore, B. C. J., Alcántara, J. I., and Glasberg, B. R. (2002). "Behavioural measurement of level-dependent shifts in the vibration pattern on the basilar membrane," Hear. Res. 163, 101-110.

Moore, B. C. J., and Glasberg, B. R. (1993). "Simulation of the effects of loudness recruitment and threshold elevation on the intelligibility of speech in quiet and in a background of speech," J. Acoust. Soc. Am. 94, 2050-2062.

Moore, B. C. J., Huss, M., Vickers, D. A., Glasberg, B. R., and Alcántara, J. I. (2000). "A test for the diagnosis of dead regions in the cochlea," Br. J. Audiol. 34, 205-224.

Moore, B. C. J., Shailer, M. J., and Schooneveldt, G. P. (1992). "Temporal modulation transfer functions for band-limited noise in subjects with cochlear hearing loss," Br. J. Audiol. 26, 229-237.

Moore, B. C. J., Wojtczak, M., and Vickers, D. A. (1996). "Effect of loudness recruitment on the perception of amplitude modulation," J. Acoust. Soc. Am. 100, 481-489.

Oxenham, A. J. (2016). "Predicting the perceptual consequences of hidden hearing loss," Trends Hear. 20, 1-6.

Patterson, R. D., and Johnson-Davies, D. (1977). "Detection of a change in the pitch of AM noise," in Psychophysics and Physiology of Hearing, edited by E. F. Evans and J. P. Wilson (Academic, London), pp. 363-371.

Plomp, R. (1983). "The role of modulation in hearing," in HearingPhysiological Bases and Psychophysics, edited by R. Klinke and R. Hartmann (Springer, Berlin), pp. 270-276.

Prendergast, G., Guest, H., Munro, K. J., Kluk, K., Leger, A., Hall, D. A., Heinz, M. G., and Plack, C. J. (2017a). "Effects of noise exposure on young adults with normal audiograms I: Electrophysiology," Hear. Res. 344, 68-81.

Prendergast, G., Millman, R. E., Guest, H., Munro, K. J., Kluk, K., Dewey, R. S., Hall, D. A., Heinz, M. G., and Plack, C. J. (2017b). "Effects of noise exposure on young adults with normal audiograms II: Behavioral measures," Hear. Res. 356, 74-86.

Robles, L., and Ruggero, M. A. (2001). "Mechanics of the mammalian cochlea," Physiol. Rev. 81, 1305-1352.

Ruggles, D., Bharadwaj, H., and Shinn-Cunningham, B. G. (2012). "Why middle-aged listeners have trouble hearing in everyday settings," Curr. Biol. 22, 1417-1422.

Schaette, R., and McAlpine, D. (2011). "Tinnitus with a normal audiogram: Physiological evidence for hidden hearing loss and computational model," J. Neurosci. 31, 13452-13457.

Schlittenlacher, J., and Moore, B. C. J. (2016). "Discrimination of amplitude-modulation depth by subjects with normal and impaired hearing," J. Acoust. Soc. Am. 140, 3487-3495.

Sergeyenko, Y., Lall, K., Liberman, M. C., and Kujawa, S. G. (2013). “Agerelated cochlear synaptopathy: An early-onset contributor to auditory functional decline," J. Neurosci. 33, 13686-13694.

Shannon, R. V., Zeng, F.-G., Kamath, V., Wygonski, J., and Ekelid, M. (1995). "Speech recognition with primarily temporal cues," Science 270, 303-304.

Shaw, E. A. G. (1974). "Transformation of sound pressure level from the free field to the eardrum in the horizontal plane," J. Acoust. Soc. Am. 56, 1848-1861.

Sheft, S., and Yost, W. A. (1990). "Temporal integration in amplitude modulation detection," J. Acoust. Soc. Am. 88, 796-805.

Stone, M. A., and Moore, B. C. J. (2014). "Amplitude-modulation detection in recreational-noise-exposed humans with near-normal hearing thresholds and its medium-term progression," Hear. Res. 317, 50-62.

Stone, M. A., Moore, B. C. J., and Greenish, H. (2008). "Discrimination of envelope statistics reveals evidence of sub-clinical hearing damage in a noise-exposed population with 'normal' hearing thresholds," Int. J. Audiol. 47, 737-750.

Valderrama, J. T., Beach, E. F., Yeend, I., Sharma, M., Van Dun, B., and Dillon, H. (2018). "Effects of lifetime noise exposure on the middle-age human auditory brainstem response, tinnitus and speech-in-noise intelligibility," Hear. Res. 365, 36-48.

Valero, M. D., Burton, J. A., Hauser, S. N., Hackett, T. A., Ramachandran, R., and Liberman, M. C. (2017). "Noise-induced cochlear synaptopathy in rhesus monkeys (Macaca mulatta)," Hear. Res. 353, 213-223.

Viana, L. M., O’Malley, J. T., Burgess, B. J., Jones, D. D., Oliveira, C. A., Santos, F., Merchant, S. N., Liberman, L. D., and Liberman, M. C. (2015). "Cochlear neuropathy in human presbycusis: Confocal analysis of hidden hearing loss in post-mortem tissue," Hear. Res. 327, 78-88.

Viemeister, N. F. (1979). "Temporal modulation transfer functions based on modulation thresholds,” J. Acoust. Soc. Am. 66, 1364-1380.

Wakefield, G. H., and Viemeister, N. F. (1990). "Discrimination of modulation depth of SAM noise," J. Acoust. Soc. Am. 88, 1367-1373.

Yeend, I., Beach, E. F., Sharma, M., and Dillon, H. (2017). "The effects of noise exposure and musical training on suprathreshold auditory processing and speech perception in noise," Hear. Res. 353, 224-236. 